\title{
Rahmenübereinkommen zur Eindämmung des Tabakgebrauchs
}

\author{
Schweizerische Gesellschaft für Prävention und Gesundheitswesen
}

Korrespondenz:

SGPG Zentralsekretariat

Effingerstrasse 54

Postfach 8172

CH-3001 Bern

Tel. 0313899286

Fax 0313899288

E-Mail: info@sgpg.ch

Internet: www.sgpg.ch
An ihrer 56. Weltgesundheitsversammlung am 21. Mai 2003 hat die WHO das Rahmenübereinkommen zur Eindämmung des Tabakgebrauchs (Framework Convention on Tobacco Control, FCTC; Internet: www.fctc.org) verabschiedet und damit einen wichtigen Meilenstein im weltweiten Kampf gegen die Folgen des Tabakkonsums gesetzt.

Das FCTC geht von der Erkenntnis aus, dass die Ausbreitung der Tabakepidemie ein weltweites Problem mit schwerwiegenden Folgen für die menschliche Gesundheit ist, welches die grösstmögliche internationale Zusammenarbeit und die Mitwirkung aller Länder bei einem wirksamen, geeigneten und umfassenden internationalen Vorgehen erfordert. Das Rahmenübereinkommen hält Ziele, Leitlinien und allgemeine Verpflichtungen für die Unterzeichnerstaaten fest sowie Massnahmen in den Bereichen «Verringerung der Nachfrage des Tabaks», «Verringerung des Angebotes von Tabak» und «Schutz der Umwelt».

Das Rahmenübereinkommen tritt in Kraft, wenn es von mindestens 40 Staaten ratifiziert wurde. Bis heute (Stand Ende März 2004) haben 101 Staaten das Rahmeneinkommen unterzeichnet und 9 ratifiziert. Die Schweiz gehört noch nicht zu den Unterzeichnerstaaten. Der letzte Termin für die Unterzeichnung ist der 29. Juni 2004.
Die Schweizerische Gesellschaft für Prävention und Gesundheitswesen bewertet das Rahmenübereinkommen als umfassende Grundlage für die öffentliche Gesundheit im Tabakbereich und unterstützt alle darin enthaltenen Elemente. An der Zentralvorstandssitzung vom 19. Februar 2004 hat sie deshalb ein Positionspapier zum FCTC verabschiedet. Darin verpflichtet sich die SGPG zu einem aktiven Einsatz für die Unterzeichnung des Rahmeneinkommens durch den Bundesrat bis 29. Juni 2004 sowie für die Ratifizierung durch das Parlament (bei einem allfälligen Referendum durch das Volk) bis spätestens 2007.

Dieser aktive Einsatz umfasst direkte Interventionen bei den zuständigen Instanzen, öffentliche Stellungnahmen zugunsten des Rahmenübereinkommens, politisches Lobbying sowie weitere unterstützende Massnahmen durch die einzelnen Mitglieder und Organe der SGPG in ihren jeweiligen Wirkungsfeldern - sei dies auf akademischer, administrativer, ziviler oder politischer Ebene.

Die SGPG ruft die FMH und alle ihr angeschlossenen Fachgesellschaften auf, sich ebenfalls für das Rahmenübereinkommen zur Eindämmung des Tabakgebrauchs zu engagieren. 